\title{
Time delay estimation for UWB non coherent receiver in indoor environment, from theory to practice
}

\author{
Abdellah Chehri ${ }^{1,4^{*}}$ D, Paul Fortier ${ }^{2}$ and Pierre-Martin Tardif ${ }^{3}$
}

\begin{abstract}
Extracting parameter estimates from noisy observations of an underlying signal is a common problem in many fields. Time delay estimation (TDE) is essential for many areas, such as localization, array processing, and radar. The performance of any estimator is often evaluated via the mean square error (MSE) that can then be compared to analytical MSE lower bounds. In this paper, we first analyze a maximum likelihood (ML) estimator based on the knowledge of noisy second order statistics of the channel. We investigate lower bounds for the time delay estimation error for ultra-wideband ranging systems operating in realistic multipath environments. Based on the Cramer-Rao lower bound (CRLB), we derive analytically a lower bound of the time delay estimation calculated using the Karhunen-Loève decomposition of the estimated channel autocorrelation matrix. Also, we investigate the practical implementation (based on energy detection) of the time delay estimator. In the second part of the paper, we have analyzed the time delay estimation performances with the energy maximization receiver. Simulations are evaluated using a simulated UWB underground mine channel. This can be considered as the first step for a global positioning system for use mining industry.
\end{abstract}

Keywords: Time delay estimation, Ultra-wideband, Non coherent receiver, Cramer-Rao lower bound, Maximum likelihood

\section{Introduction}

Ultra-wideband (UWB) is a promising technique in the application of short-range high-speed wireless communication, radar, and precise location. UWB signal is capable to provide an accurate ranging, owing to its inherent high delay resolution and ability to penetrate obstacles. Therefore, it is the technology of choice for localization systems, particularly in densely cluttered environments.

Time delay estimation using UWB transmission is currently the most popular technique for accurate ranging and tracking. In the literature, several methods for UWB signal time delay estimation can be found in [1-3]. Examples of low complexity estimators include a maximum peak detection method and a threshold detection method. In the paper, a threshold is chosen a priori, and

\footnotetext{
* Correspondence: achehri@uqac.ca

${ }^{1}$ University Ottawa, 800 King Edward Avenue, Ottawa, ON K1N 6N5, Canada ${ }^{4}$ Department of Applied Sciences, University of Quebec in Chicoutimi (UQAC), 555, boul. de l'Université, Chicoutimi, QC G7H 2B1, Canada Full list of author information is available at the end of the article
}

the time delay estimation is defined as the instant when the received amplitude goes above this threshold [4]. In spite of its simplicity, this method works quite well for UWB signals, especially in high signal-to-noise ratio (SNR) environments.

Other time delay estimation techniques are studied for use in multipath channels not necessary with a high SNR, including the maximum-likelihood technique. Indeed, the time delay estimation in a multipath environment is closely related to channel estimation, where channel amplitudes and time of arrivals are jointly estimated using, for example, a maximum likelihood (ML) approach [5]. However, what is the meaning of the lower bound of the time delay estimation (TDE) estimation when the received signal is completely or partially unknown?

In fact, the problem of detecting an unknown signal through a channel containing additive Gaussian noise has received attention for several years. Although this topic is old, there continues to be progress and several problems are investigated recently particularly in the areas of networks synchronization [2], ranging [3] and 
localization applications [6]. The maximum likelihood (ML) estimator is a widely used method for estimation problems. The Cramer-Rao lower bound is a general lower bound on the minimum estimation of a random parameter [7]. It has been widely used to lower bound the MSE of symbol timing estimators [2, 5, 8]. However, the valid application of this bound requires that the estimator be unbiased. The measure in which we are interested in is the mean-square error of the estimate.

Let us assume access to an observation $\mathrm{Z}$ that has probability density function (pdf) $p(Z ; x)$, meaning that the pdf depends on a parameter vector $\mathrm{x}$ to be estimated. Let us assume the existence of an unbiased estimator $\hat{x}(Z)$, meaning that we have:

$$
E\{\hat{x}(Z)\}=x
$$

in which $E$ denotes expectation parameterized by $x$. Then, under fairly broad regularity conditions, the Cramer-Rao lower bound (CRLB) is

$$
E\left\{[\hat{x}(Z)][\hat{x}(Z)]^{T}\right\} \geq J^{-1}
$$

Where

$$
J=E\left\{\left[\nabla_{x} \log (p(Z ; x))\right]\left[\nabla_{x} \log (p(Z ; x))\right]^{T}\right\}
$$

The remainder of this paper is organized as follows. Section 2 defines the mathematical model of the ML estimator. We define the Cramer-Rao lower bound of the estimator in Section 3. Section 4 describes a practical version of the ML estimator by using an energy detection receiver. Section 5 is devoted to results and discussion. A conclusion is given in Section 6.

\section{Maximum likelihood estimator of time delay}

The transmitted signal $s(t)$ is given by

$$
s(t)=\sqrt{E_{t x}} p(t)
$$

Here, $E_{t x}$ is the transmission energy and $p(t)$ has unit energy and finite duration $T_{p}$. The signal is transmitted through the multipath channel $h(t)$, with propagation time $\delta$, so the received signal is described by:

$$
r(t)=\sqrt{E_{r x}} h(t-\delta)+n(t)
$$

where $E_{r x}$ is the received signal energy incorporating the transmission energy and the total channel power gain. We assume $\delta \in\left[0, T_{\mathrm{f}}\right]$, and assume $T_{f}$ is known a priori. $n(t)$ is an additive white Gaussian noise (AWGN), modeled as a zero-mean complex circularly symmetric Gaussian process with power spectral density $N_{0} / 2 . h(t)$ is the overall channel response (assumed to be statistically independent of $n(t))$ and is given by:

$$
h(t)=\sum_{l=0}^{L-1} \alpha_{l} \delta\left(t-\tau_{l}\right)
$$

The attenuation values $\left(\alpha_{l}\right)$ and the delays $\left(\tau_{l}\right)$ vary with time in a way that depends on the physical environment. In fact, it is usually difficult, if not impossible, to estimate these parameters perfectly. However, the variation of the attenuation and delay fluctuations are sufficiently independent statistically, hence the Central Limit Theorem can be applied. Therefore, the received signal of (1) can be described as a sample function from an approximately Gaussian process with a mean and autocorrelation function.

Without loss of generality, when UWB single pulse is transmitted into a multipath channel, the received signal can be represented as follows (see Fig. 1):

$$
r(t)=s(t ; \delta)+n(t)
$$

where $\delta$ is the time delay to be estimated. $\delta$ can be modeled in two different ways. In the first, we assume that $\delta$ is a nonrandom parameter and we use maximum likelihood estimation procedures. In the second, we assume that $\delta$ is the value of a random variable with a known probability density $p(\delta)$. For random parameters, we can use Bayes estimates with various cost functions. We shall confine our discussion to maximum a posteriori estimator (MAP) estimates.

Now the problem is how to estimate the unknown parameter $\delta$ for any given $r(t)$, where $\delta \in\left[0 ; T_{f}\right]$.

The particular estimates we shall be concerned with here are the maximum-likelihood estimates. We now turn

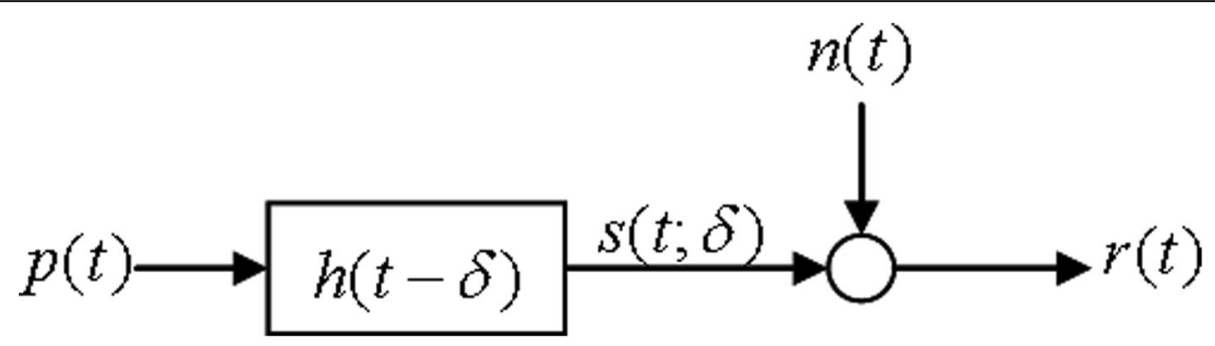

Fig. 1 System model with represented delay 
to a derivation of the pertinent maximum-likelihood estimates and a discussion of their physical meaning.

In order to find the maximum likelihood (ML) estimate of $\delta$, we can represent the received signal into two components (Fig. 2), one to present the signal in a short window, the second represents the remainder of the signal.

We begin by holding the unknown parameters fixed and making a Karhunen-Loève [7] expansion of the received signal $r(t), 0 \leq t \leq T_{f}$ in terms of the eigenfunctions of its two components autocorrelation function.

$$
R_{r}(t, u: \delta)= \begin{cases}R_{s}(t, u: \delta)+\frac{N_{0}}{2} \delta(t, u) & \rightarrow r_{1}(t) \\ \frac{N_{0}}{2} \delta(t, u) & \rightarrow r_{2}(t)\end{cases}
$$

We note that the signal $\left\{r_{1}(t)\right\}$ is the part of received signal projected in the basis $\phi_{k}(t)$ in the interval $[\delta ; \delta+$ $\left.T_{d}\right]$. The first component $\left\{r_{1}(t)\right\}$ is calculated with:

$$
r_{1}(t)=\sum_{k=0}^{m} r_{1, k} \phi_{k}(t)
$$

where

$$
r_{1, k}=\int_{\delta}^{\delta+T_{d}} r(t) \phi_{k}(t) d t
$$

we define

$$
\begin{aligned}
\int_{\delta}^{\delta+T_{d}}\left(R_{s}(t, u: \delta)\right. & \left.+\frac{N_{0}}{2} \delta(t, u)\right) \phi_{i}(t) d t \\
& =\left(\lambda_{i}+\frac{N_{0}}{2} \delta(t, u)\right) \phi_{i}(t)
\end{aligned}
$$

and

$$
\int_{\delta}^{\delta+T_{d}} \phi_{i}(t) \phi_{k}(t) d t=\delta_{i k}
$$

which means that all functions are orthogonal. The $\left\{r_{1, k}\right.$, $\left.\phi_{k}(t)\right\}$ are all functions of the unknown parameters $\delta$. For easy writing, this dependence is not indicated. Under the hypotheses of the problem, the $\left\{r_{1, k}\right\}$ are Gaussian random variables satisfying:

$$
\begin{gathered}
E\left\{r_{1, k}\right\}=0 \\
E\left\{\left(r_{1, k}\right)\left(r_{1, i}\right)\right\}=\left(\lambda_{i k}+\frac{N_{0}}{2}\right)
\end{gathered}
$$

The joint probability density for the first $m$ terms of $\left(r_{1}, k\right)$ can now be written down explicitly. For the case "signal + noise" we have:

$$
\begin{aligned}
P_{s}\left(r_{1,1}, r_{1,2} \cdots, r_{1, m} / \delta\right)= & \prod_{i=1}^{m} \frac{1}{\sqrt{2 \pi\left(\lambda_{i}+\frac{N_{0}}{2}\right)}} \cdot \exp \\
& \left(-\frac{1}{2} \sum_{i=1}^{m} \frac{r_{1, i}^{2}}{\left(\lambda_{i}+\frac{N_{0}}{2}\right)}\right)
\end{aligned}
$$

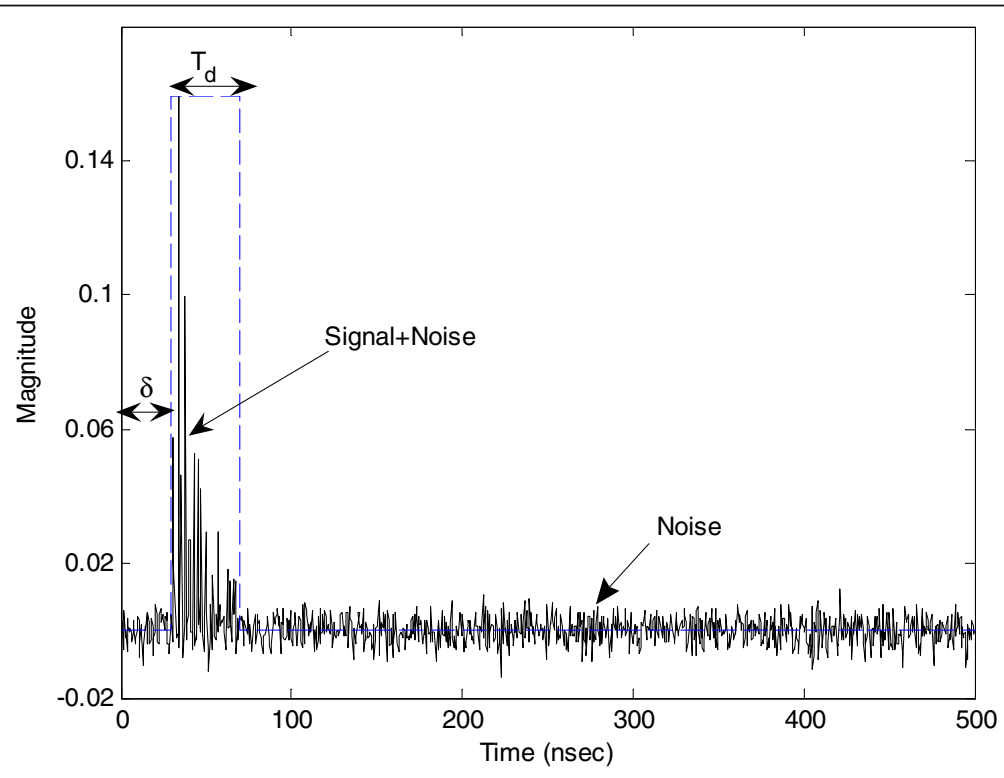

Fig. 2 Example of received signal represented by two components (signal + noise and only noise) 
The second component $\left(r_{2, k}\right)$ is define in the interval $D$, where $D=[0, \delta] \cup\left[\delta+T_{d}, T_{f}\right]$. It was projected into another orthogonal basis $\left\{\mu_{i} ; \phi_{i}(t)\right\}$

$$
\begin{gathered}
\int_{(t, u) \in D} R_{b}(t, u) \phi_{k}(u) d u=\mu_{k} \phi_{k}(t) \\
\int_{t \in D} \phi_{k}(t) \phi_{i}(t) d t=\delta_{i k}
\end{gathered}
$$

and

$$
r_{2}(t)=\sum_{k=0}^{m} r_{2, k} \phi_{k}(t)
$$

The joint probability density for the first $m$ terms of $\left(r_{2, k}\right)$, the case "noise only", is given by:

$$
\begin{aligned}
P_{n}\left(r_{1,1}, r_{1,2} \cdots, r_{1, m} / \delta\right)= & \prod_{i=1}^{m} \frac{1}{\sqrt{2 \pi\left(\frac{N_{0}}{2}\right)}} \cdot \exp \\
& \left(-\frac{1}{2} \sum_{i=1}^{m} \frac{r_{2, i}^{2}}{\left(\frac{N_{0}}{2}\right)}\right)
\end{aligned}
$$

with

$$
\begin{gathered}
r_{1}(t)=\int_{\delta}^{\delta+T_{d}} r(t) \phi_{i}(t) d t \\
r_{2}(t)=\int_{0}^{\delta} r(t) \phi_{i}(t ; \delta) d t+\int_{\delta+T_{d}}^{T_{f}} r(t) \phi_{i}(t ; \delta) d t
\end{gathered}
$$

and

$$
\begin{gathered}
r_{1, i}=s_{i}+n_{1, i} \\
r_{2, i}=n_{2, i}
\end{gathered}
$$

The joint probability of the two components of the received signal $\{r(t)\}$ is:

$$
\Lambda(r)=P\left(\left\{r_{1, i}\right\},\left\{r_{2, i}\right\}\right)=P\left(\left\{r_{1, i}\right\}\right) \times P\left(\left\{r_{2, i}\right\}\right)
$$

The likelihood ratio can be written as follows:

$$
\frac{\Lambda(r)}{R}=\frac{P\left(\left\{r_{1, i}\right\}\right) \times P\left(\left\{r_{2, i}\right\}\right)}{R}
$$

where

$$
R=\frac{1}{2 \pi\left(\frac{N_{0}}{2}\right)} \prod_{i=1}^{m} \cdot \exp \left(-\frac{1}{2} \sum_{i=1}^{m} \frac{r_{1, i}^{2}+r_{2, i}^{2}}{\left(\frac{N_{0}}{2}\right)}\right)
$$

We suppose that $R$ is independent of the estimate parameter $\delta$. After simplification, we obtain:

$$
\begin{aligned}
L & =\frac{\Lambda(r)}{R} \\
& =\prod_{i=1}^{m} \sqrt{\frac{\frac{N_{0}}{2}}{\left(\lambda_{i}+\frac{N_{0}}{2}\right)}} \cdot \exp \left(-\frac{1}{2} \sum_{i=1}^{m} \frac{\lambda_{i} r_{1, i}^{2}}{\frac{N_{0}}{2} \cdot\left(\lambda_{i}+\frac{N_{0}}{2}\right)}\right)
\end{aligned}
$$

The quantity $L$ given in (6) is a function of the received signal on $0 \leq t \leq T_{f}$ and the assumed value of $\delta$. The estimated value $\hat{\delta}$ is defined to be the value of $\delta$ that maximizes the likelihood ratio $\mathrm{L}$. Then the logarithm $\mathrm{l}=$ $\log (\mathrm{L})$ is written as follows:

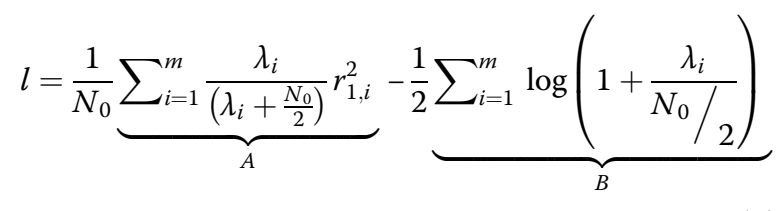

We shall now proceed to interpret the maximization of (7) in physical terms. The first term A appearing in (7) can be written directly in terms of $r(t)$ by making use of (3)

$$
\begin{aligned}
\sum_{i=1}^{m} \frac{\lambda_{i}}{\left(\lambda_{i}+\frac{N_{0}}{2}\right)} r_{1, i}^{2} & =\iint_{\delta}^{\delta+T_{d}} r(t) r(u) \\
& {\left[\sum_{i=1}^{m}\left(\frac{\lambda_{i}}{\left(\lambda_{i}+\frac{N_{0}}{2}\right)}\right)\right] \phi_{k}(t) \phi_{k}(u) d t d u } \\
& =\int_{\delta}^{\delta+T_{d}} r(t) h(t, u) r(u) d t d u
\end{aligned}
$$

where $h(t ; u)$ satisfies the integral equation

$$
\int_{\delta}^{\delta+T_{d}} R_{r}(t, u) h\left(t^{\prime}, u\right) d t^{\prime}=R_{s}\left(t^{\prime}, u\right)
$$

or in terms of eigenfunctions and eigenvalues

$$
h(t, u)=\sum_{i=1}^{m} \frac{\lambda_{i}}{\left(\lambda_{i}+\frac{N_{0}}{2}\right)} \phi_{i}(t) \phi_{i}(u)
$$

The integral of (9) is analyzed on the theory of least mean square filtering. It is a trivial matter to verify that $h(t ; u)$ is the impulse response of the linear time-varying filter (channel) that provides the best (in the sense of minimum mean square error) estimate of $s(t)$ given by: 


$$
r(t)=s(t)+n(t) \quad 0 \leq t \leq T_{f}
$$

In symbols, $h(t ; u)$ minimizes

$$
E\left[s(t)-\int_{0}^{T_{f}} h(t, u) r(u)\right]^{2}
$$

The physical interpretation of the term $A$ is given as

$$
\begin{aligned}
\sum_{i=1}^{m} \frac{\lambda_{i}}{\left(\lambda_{i}+\frac{N_{0}}{2}\right)} r_{1, i}^{2} & =-\frac{1}{N_{0}} \int_{0}^{T_{f}} h(t, u) \\
& {\left[\int_{0}^{T_{f}} h(t, u) r(u) d u\right] d t }
\end{aligned}
$$

Equation (11) represents the correlation between the received signal $r(t)$ and its best estimate in the sense of linear least mean square. Equality (9) can be solved using some numerical methods (such as Fredholm integral). We shall note that the solution of (11) can also be reduced to the solution of a certain Wiener-Hopf type of integral Eq. (7).

The second term of (7) (i.e., B) makes the ML estimator biased. The convergence follows easily

$$
\begin{array}{r}
\sum_{i=1}^{m} \log \left(1+\frac{\lambda_{i}}{N_{0} / 2}\right) \leq \sum_{i=1}^{m} \frac{2 \lambda_{i}}{N_{0}} \\
=\frac{2}{N_{0}} \int_{0}^{T_{f}} R_{s}(t, t) d t
\end{array}
$$

The integral is just the excepted value of the energy in the process, which was assumed to be finite.

We now rewrite the maximum likelihood function by using (7) and (8) to obtain

$$
l=\frac{1}{N_{0}} \iint r(t) h(t, u) r(u) d t d u-\frac{1}{2} \sum_{i=1}^{m} \log \left(1+\frac{2 \lambda_{i}}{N_{0}}\right)
$$

The realization of the maximum likelihood time delay estimator is shown in Fig. 3. The estimated value corresponds to the maximum value of ML. Even though the procedure is well defined, the actual implementation is difficult. To illustrate this, we consider the case of the maximum likelihood estimation of a parameter $\delta$. We assume that it lies in the interval $[0 ; T f]$. We divide the parameter range into intervals of length $\Delta$. The center points of these intervals are:

$$
\begin{gathered}
\delta_{1}=\frac{\Delta}{2} \\
\delta_{2}=\delta_{1}+\frac{\Delta}{2}
\end{gathered}
$$

and so forth, etc. Therefore, there are $M$ intervals. We then construct ML estimator, $(i=1 ; \cdots ; M)$ by using the parallel processing shown in Fig. 3. However, some remarks are worthwhile; first, we have to solve a different integral equation to find the filter in each path. Thus, the estimation problem has the same degree of complexity as an $\mathrm{M}$-ary detection problem in the sense that we must build M-parallel processors. Also, we have to

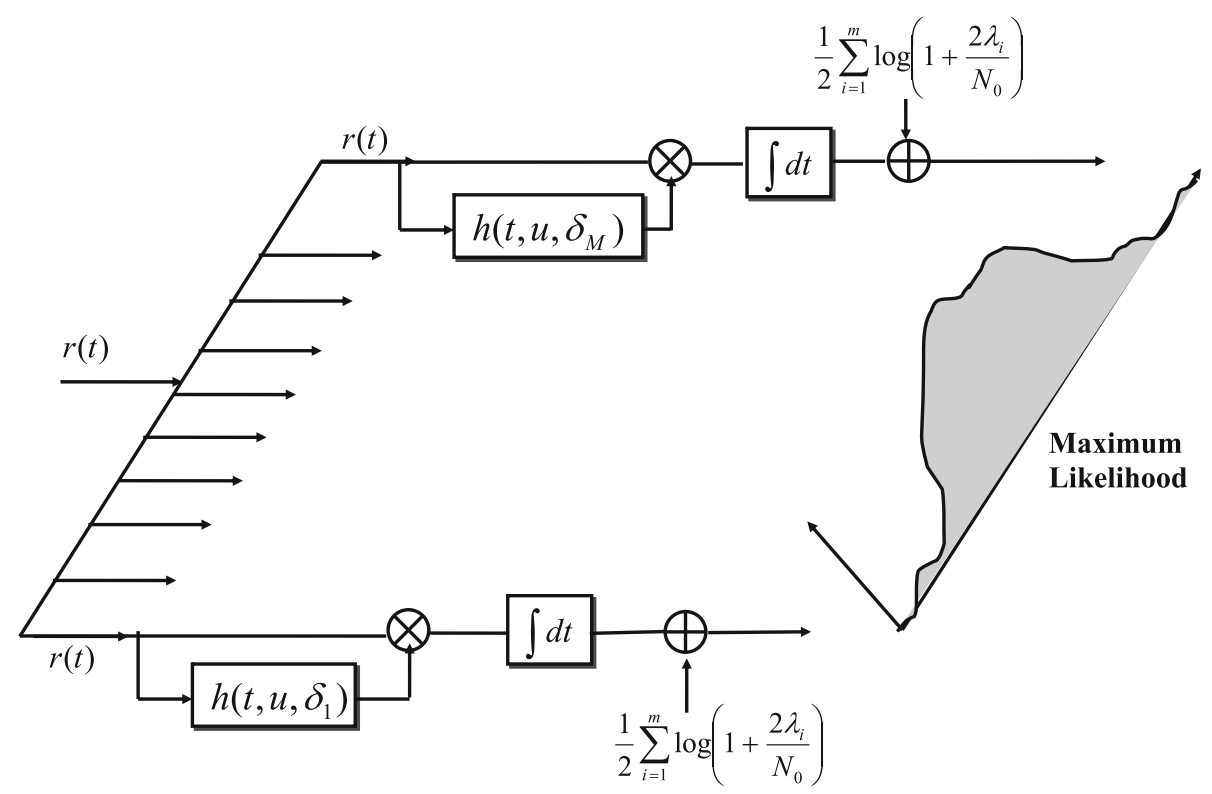

Fig. 3 Generation of maximum likelihood for TD estimation 
consider the effect of the grid size $\Delta$ which is taken as a trade-off between accuracy and complexity.

\section{Lower bound on the variance estimation}

We shall begin by attempting to find the value of $\delta$ that maximizes the likelihood ratio. This is equivalent to finding the value of $\delta$ maximizing (13)

$$
\frac{\partial l}{d \delta}=X_{1}+X_{2}
$$

Differentiating the first term, we obtain:

$$
X_{1}=\frac{1}{N_{0}} \iint r(t) \frac{\partial h(t, u)}{d \delta} r(u) d t d u
$$

We have seen that $h(t ; u)$ is the optimal filter. With this filter, we can observe and make the mean square error estimate of $s(t ; \delta)$ as in the detection problem. We use the inverse kernel $Q(t ; u)$, which can be written as follows:

$$
Q(t, u)=\frac{2}{N_{0}}[\delta(t, u)-h(t, u)]
$$

Using (15) and (16) we can find

$$
\begin{aligned}
X_{1} & =\frac{1}{N_{0}} \iint r(t) \frac{\partial h(t, u)}{d \delta} r(u) d t d u \\
& =-\frac{1}{2} \iint r(t) \frac{\partial Q(t, u)}{d \delta} r(u) d t d u
\end{aligned}
$$

For differentiating the second term, we must represent the series as a continued function

$$
\frac{1}{2} \sum_{i=1}^{m} \log \left(1+\frac{2 \lambda_{i}}{N_{0}}\right)=-\frac{1}{N_{0}} \int \xi(t) d t
$$

$\xi(t)$ is the mean square filtering error developed in [7]. Thus, we can calculate the differentiating of the second term of (14) by using (18). We obtain:

$$
X_{2}=-\frac{1}{N_{0}} \int \xi(t) d t \frac{1}{2} \iint R_{r}(t) \frac{\partial Q(t, u)}{d \delta} d t d u
$$

Putting (17) and (19) into (14)

$$
\begin{aligned}
\frac{\partial l}{d x} & =-\frac{1}{2} \iint r(t) \frac{\partial Q(t, u)}{d \delta} r(u) d t d u \\
& +\frac{1}{2} \iint R_{r}(t) \frac{\partial Q(t, u)}{d \delta} d t d u
\end{aligned}
$$

To find the lower bound on the variance of $\delta$ estimation, we must calculate the second derivate of (14)

$$
\operatorname{Var}[\hat{\delta}-\delta] \geq-\left\{E\left[\frac{\partial^{2} l}{d \delta^{2}}\right]\right\}^{-1}
$$

Using (20)

$$
\operatorname{Var}[\hat{\delta}-\delta] \geq-\left\{-\frac{1}{2} \iint \frac{R_{r}(t, u)}{d \delta} \frac{\partial h(t, u)}{d \delta} d t d u\right\}^{-1}+\rho
$$

where $\rho$ is a terms whose expectation is zero. We note

$$
J=-\frac{1}{2} \iint \frac{R_{r}(t, u)}{d \delta} \frac{\partial h(t, u)}{d \delta} d t d u
$$

Using (10), we can write $J$ in terms of eigenfunctions and eigenvalues. Differentiating $R_{r}(t, u), h(t, u)$, we obtain:

$$
\begin{aligned}
J(\delta) & =\frac{1}{2} \sum_{i=1}^{m}\left(\frac{\left[\partial \lambda_{i} / d \delta\right]}{\lambda_{i}+\frac{N_{0}}{2}}\right)^{2} \\
& +\frac{2}{N_{0}} \sum_{i=1}^{m}\left(\frac{\lambda_{I}^{2}}{\lambda_{i}+\frac{N_{0}}{2}} b_{i}(\delta)\right) \\
& -\frac{2}{N_{0}} \sum_{i=1}^{m} \sum_{j=1}^{m}\left(\frac{\lambda_{i} \lambda_{j}}{\lambda_{i}+\frac{N_{0}}{2}} a_{i j}(\delta)\right)
\end{aligned}
$$

where

$$
\begin{aligned}
& b_{i}(\delta)=\int\left(\frac{\partial \phi_{i}(t)}{d \delta}\right)^{2} d t \\
& a_{i j}(\delta)=\int\left(\frac{\partial \phi_{i}(t)}{d \delta}\right) \phi_{j}(t) d t
\end{aligned}
$$

By developing (25) and (26)

$$
\begin{aligned}
b_{i}(\delta) & =\int_{\delta}^{\delta+T_{d}}\left(\frac{\partial \phi_{i}(t)}{d \delta}\right)^{2} d t=\int_{0}^{T_{d}}\left(\frac{\partial \phi_{i}(t-\delta)}{d \delta} / \delta=0\right)^{2} d t \\
& \left.=\int_{0}^{T_{d}}\left(\frac{\partial \phi_{i}(u)}{d u}\right]_{u=t-\delta} / \delta=0\right)^{2} d t=\int_{0}^{T_{d}}\left(\frac{\partial \phi_{i}(u)}{d u}\right)^{2} d t
\end{aligned}
$$

And

$$
\begin{aligned}
a_{i j}(\delta) & =\int_{\delta}^{\delta+T_{d}}\left(\frac{\partial \phi_{i}(t ; \delta)}{d \delta}\right) \phi_{j}(t ; \delta) d t \\
& =\int_{0}^{T_{d}}\left(\left(-\frac{\partial \phi_{i}(t-\delta)}{d \delta}\right) \phi_{j}(t-\delta) / \delta=0\right) d t \\
& =\int_{0}^{T_{d}}\left(\left.\left(-\frac{\partial \phi_{i}(u)}{d u}\right) \phi_{j}(u)\right|_{u=t-\delta} / \delta=0\right) d u \\
& =-\int_{0}^{T_{d}}\left(\frac{\partial \phi_{i}(u)}{d u}\right) \phi_{j}(u) d u
\end{aligned}
$$

In (24), we suppose that $\lambda_{i}$ are not function of the delay $\delta$ (without loss of generality and to avoid heavy notations we take $\delta=0$ ), thus 


$$
\begin{aligned}
J(\delta) & =\frac{2}{N_{0}} \sum_{i=1}^{m}\left(\frac{\lambda_{I}^{2}}{\lambda_{i}+\frac{N_{0}}{2}} b_{i}(\delta)\right) \\
& -\frac{2}{N_{0}} \sum_{i=1}^{m} \sum_{j=1}^{m}\left(\frac{\lambda_{i} \lambda_{j}}{\lambda_{i}+\frac{N_{0}}{2}} a_{i j}(\delta)\right)
\end{aligned}
$$

For simplicity, we shall consider only unbiased estimates in which case the Cramer-Rao lower bound on the variance of any estimate $\hat{\delta}$ of $\delta$ is written in the form

$$
\operatorname{Var}[\hat{\delta}-\delta]^{2} \geq \frac{1}{J(\delta)}
$$

To evaluate the numerical value of $J(\delta)$ given in Eq. (28), we assume the knowledge of the second order of the channel $R_{r}(t, u)$ characterized by a finite number of eigen-modes $\left(\lambda_{i}\right)$. In Fig. 4, we plot the obtained lower bound on the root mean square error (RMSE) for different delay spread durations $\mathrm{Td}$. The pulse duration is $T_{p}=1 \mathrm{~ns}$, the observation period is of length $T_{f}=100 \mathrm{~ns}$. From the figure, we observe three different regions:

- Region 1: the full ambiguity region corresponding to a very small SNR; in this region, the receiver sees the signal as noise and the error, in this case, is uniformly distributed over the a priori interval.

- Region 2: the second region is similar to the Cramer-Rao region. This region corresponds to a high SNR. In this case, the receiver success to match well the signal with very small uncertainty. We observe also that with increasing of delay spread $T_{d}$ the error variance is reduced.

- Region 3: The threshold or intermediate region is located between the two previous regions. The estimation error in these cases exceeds the CRLB by a large factor and describes more precisely the limit of the estimation error. This region is more realistic bound, especially for UWB systems that are supposed to operate in this range of SNR.

\section{Method}

The configuration of the maximum likelihood time delay estimator presented in Fig. 3 is based on the optimum unrealizable filter (noncausal) and requires the solution of a Fredholm integral Eq. (9). Hence, the optimal estimatorcorrelator, considered in Section (2) for many reasons, is impractical. Other configurations that eliminate the unrealizability problem are proposed by Van Trees [7] (i.e., filter-correlator receiver, filter-squarer receiver). Due to the lack of space, these analyses are not presented in this paper. Moreover, in a multipath environment, the correlation output needs to be maximized over a very large dimensional space. Therefore, the complexity of the time delay estimator in the optimal receiver is very difficult. However, more practical time delay estimation algorithms have recently been proposed, the most often used is based on an energy detection receiver due to its low implementation complexity [9-20].

Compared with coherent receivers (optimal receiver), the energy detection (ED) receiver is a suboptimal but practical and widely used receiver. The energy detection receiver is a

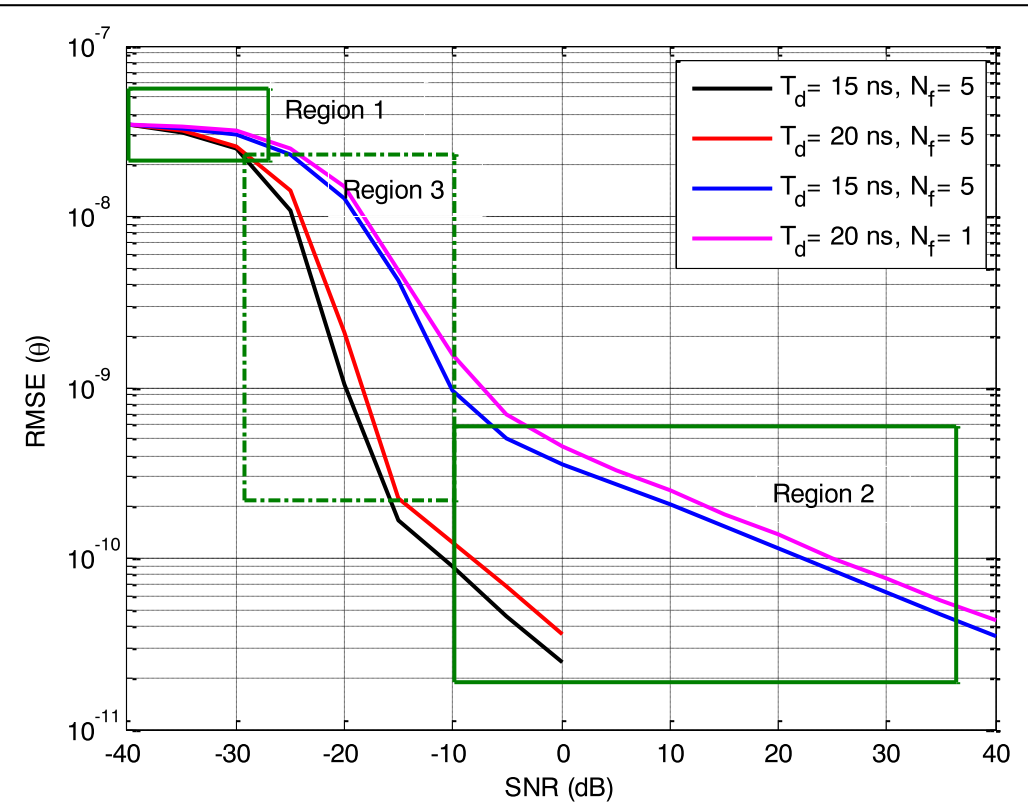

Fig. 4 Lower bound for single and multi-frame estimators vs, SNR (Td=15 ns, 20 ns) 
feasible solution for time delay estimation in ultra-wideband ranging. The task of ED estimation under UWB multipath channel is to determine the first path (FP). In the ED receiver, threshold-crossing (TC) could be also used. For example, Fig. 5 shows the block diagram of the discrete time energy detection receiver (ED). The received signal is first passed through a band-pass filter (BPF) to reduce the noise power. After low noise amplification, the signal is squared and then passed to a block of integrators that integrate the received signal in different time slots. The output of the integrator is then sampled at rate $\Delta$. The produced samples will act as the test statistics of the time delay estimator. The advantage of this receiver scheme is that it is relatively easy in implementation when compared with the previous estimator scheme (Fig. 3).

For what follows, we assume that the receiver has acquired the sequence of the desired users. We are interested in estimating the time delay, $\delta$, of the direct path with the corresponding user based on observations of the received signal $r(t)$. Also, we assume that the signal always arrives within one frame duration $\left(\delta<T_{f}\right)$, so no inter-frame interference (IFI) are considered.

The observed signal forms the input to the ED, whose output is sampled at every $\Delta$ seconds (i.e., step of energy detection), thus $k=\left\llcorner\left(T_{f}-T_{i n t}\right) / \Delta\right\rfloor$ is the number of window (i.e., block) used for the time delay estimation. Energy samples could be obtained by sampling the signal after a square-law device and an integrator with a duration of $T_{\text {int }}$ seconds by using the following equation

$$
\mathrm{z}[k]=\int_{k \Delta}^{k \Delta+T_{\text {int }}}|r(t)|^{2} d t, \quad k=0,1, \cdots, K-1
$$

Based on the observation of $\mathrm{z}=\left[z_{o}, z_{1}, \cdots, z_{K-1}\right]$, the receiver estimates the value of $\delta$ which is given by:

$$
\underbrace{\hat{\delta}=\operatorname{argmax}\{z[k]\}}_{0 \leq k \leq K-1}
$$

In other words, the block with the largest signal energy is selected.

\section{Results and discussion}

To investigate the UWB ranging system for an ad hoc network in an underground mine environment, a custom-made simulation tool was developed. In order to simulate the positioning scheme involving these nodes, it is necessary to establish a UWB-based network from scratch. Each of the nodes needs to be able to transmit and receive UWB signals. In addition to transmitters and receivers, a channel model will have to be simulated in order to obtain results that are as realistic as possible.

In fact, the simulations based on realistic channel modeling allow us to evaluate the performance of the energy detection (ED) receiver without the need for expensive hardware and real-time software implementation. A TD estimator scheme can then be implemented to test the accuracy under satisfactory conditions.

The propagation aspects of the wireless channel were modeled using the underground mine UWB-channel. The channel realizations are sampled at $3 \mathrm{GHz}$; for each channel realization, a time delay uniformly distributed with $\left(0, T_{f}\right)$ is generated, where $T_{f}=100 \mathrm{~ns}$. The data rate is $2 \mathrm{Mbps}$ and each bit consists of only one pulse.

The proposed channel model can be used for generating time series which can be used for successfully designing robust industrial UWB-based wireless sensor networks (WSNs), and for simulating the performance of WSNs in harsh industrial environments.

The path loss exponent is equal to $\eta=1.47$ for line of sight (LOS) and 2.45 for NLOSS, respectively. The

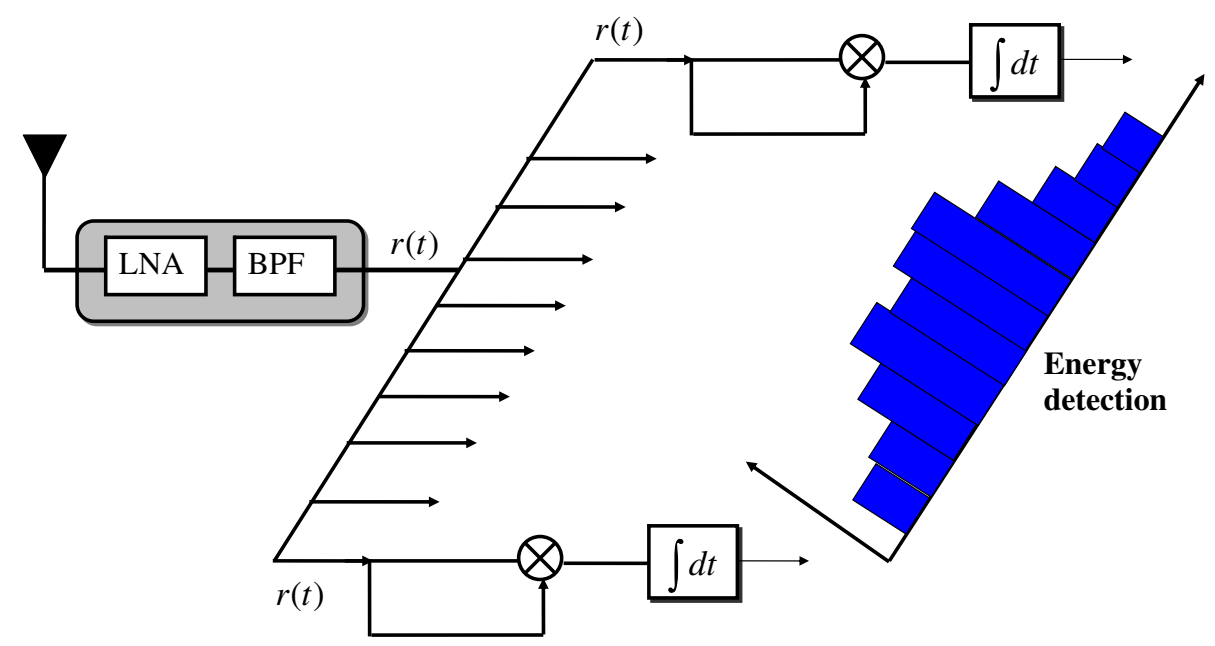

Fig. 5 Block diagram of energy detection receiver for time delay estimation 


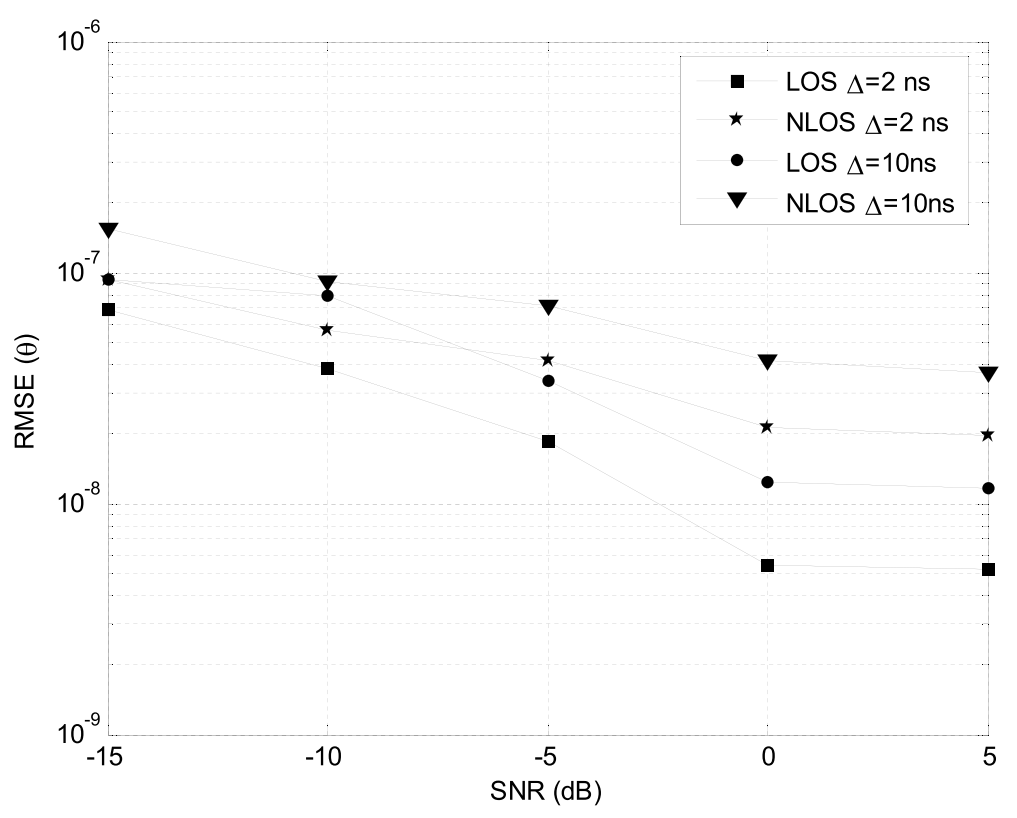

Fig. 6 RMSE of TD energy detection estimator vs. SNR, for various integrating step $\Delta$

mean standard deviation of shadow fading (in $\mathrm{dB}$ ) was assumed to be $\sigma_{s}=1.1$ for LOS and $\sigma_{s}=2.94$ for NLOS, respectively. Other details about the UWB channel parameters in an underground mine are given in [21].

The monocycle pulse, modeled as a second derivate Gaussian waveform with width $T_{p}=2 \mathrm{~ns}$, is considered for all scenarios, and it is convolved with the realizations of UWB channels to obtain the transmitted signal $s(t)$.
An additive white Gaussian noise is added to the transmitted signal to obtain the received signal $r(t)$.

The simulation investigated the estimation of the time delay for a signal between transmitter and receiver using discrete time estimation by energy maximization. First, the performance in terms of root mean squared error is evaluated by simulations for different scenarios. The sensitivity of the estimated time delay with respect to the SNR is evaluated.
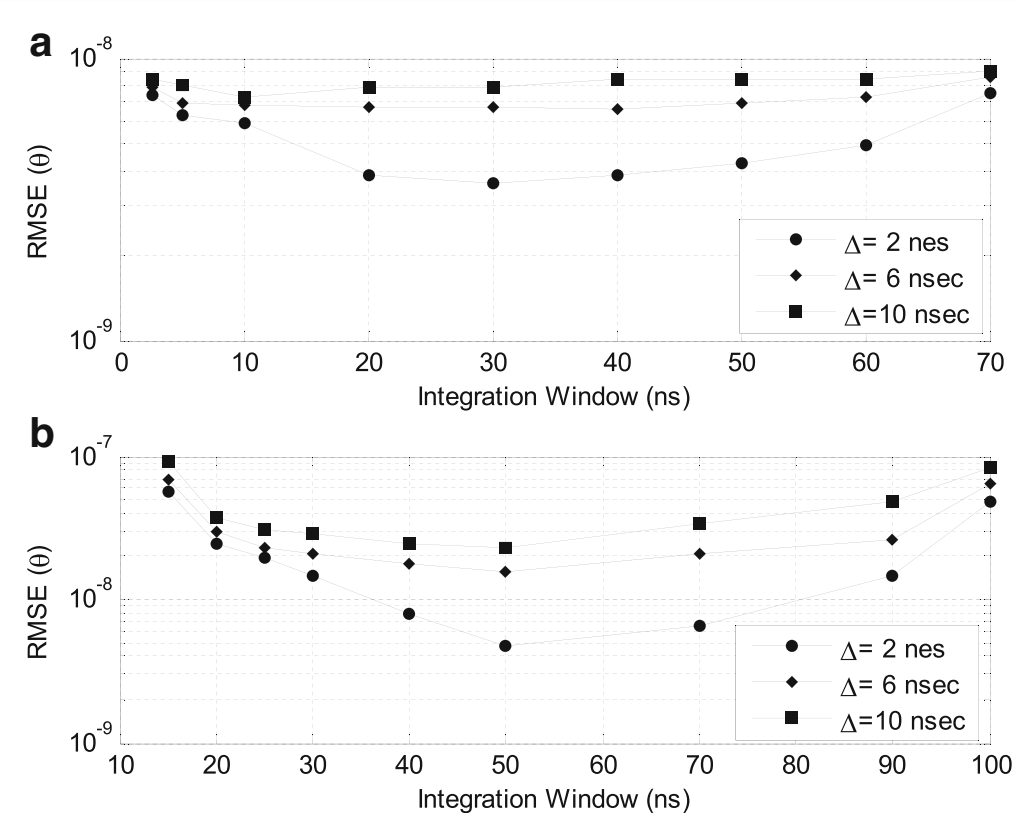

Fig. 7 RMSE of TD energy detection estimator vs. integration window length Tint, for various integrating step $\Delta$, SNR $=-10 \mathrm{~dB}$, a $L O S$ case $\mathbf{b}$ NLOS case 


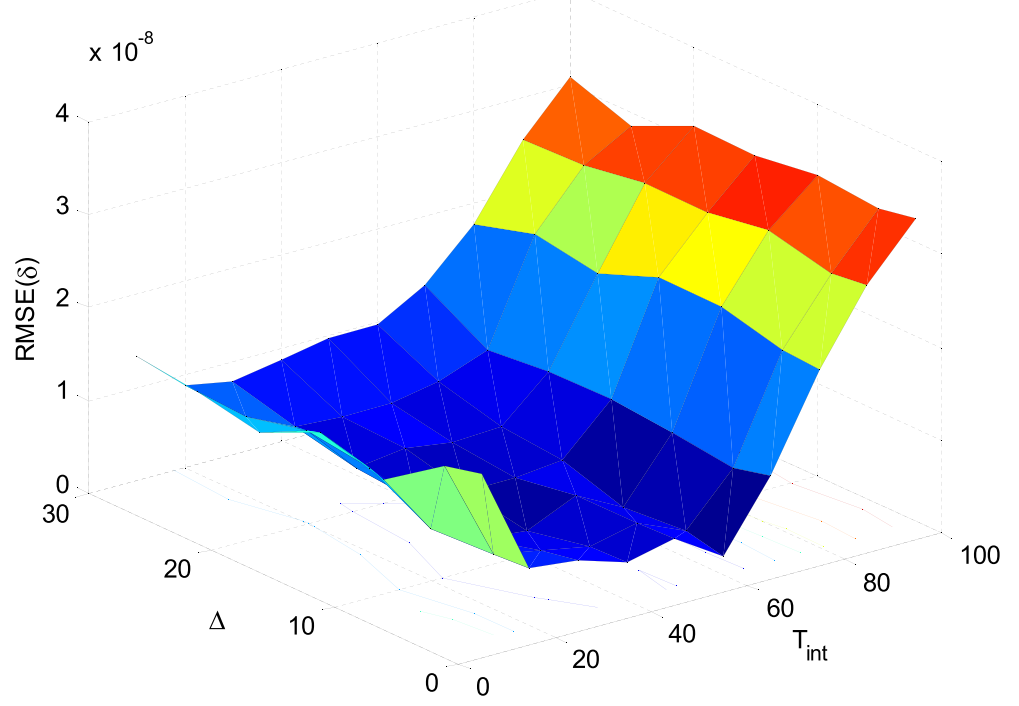

Fig. 8 RMSE of TD energy detection estimator vs. integration window length Tint and integrating step $\Delta$, for LOS scenario

In Fig. 6, we plot the obtained lower bound of the root mean square error (RMSE) for a fixed integration duration $T_{\text {int }}$ (i.e., $30 \mathrm{~ns}$ for both LOS and NLOS) vs. average transmitted SNR. We can see that as the SNR increases, we can easily notice that the RMSE of time delay is reduced. Accordingly, the proposed method has a superior capability of UWB ranging even in condensed and harsh industrial environments.

Given the last result, we now look at the impact of the configuration between transmitter and receiver (i.e., LOS and NLOS) on the choice of the integration window length. Figure 7 illustrates the RMSE of energy maximization estimator vs the integration window length Tint, for various discretization step $\Delta$, and $S N R=-10 \mathrm{~dB}$.
We observe a trade-off behavior where the optimal time integration depends on the configuration (LOS or NLOS). Indeed, for $T_{i n t}$ greater than the optimal window length, the loss is due to the fact that the integrator collects no more significant signal components. While in the contrary case, the integrator misses significant signal components. These analyses are also shown for varying both $T_{i n t}$ and $\Delta$ for LOS (Fig. 8) and NLOS (Fig. 9).

\section{Conclusion}

This paper is focused on time delay estimation for UWB impulse radio. In the first part, we have derived a maximum likelihood (ML) estimator based on the knowledge of noisy second order statistics of the received signal.

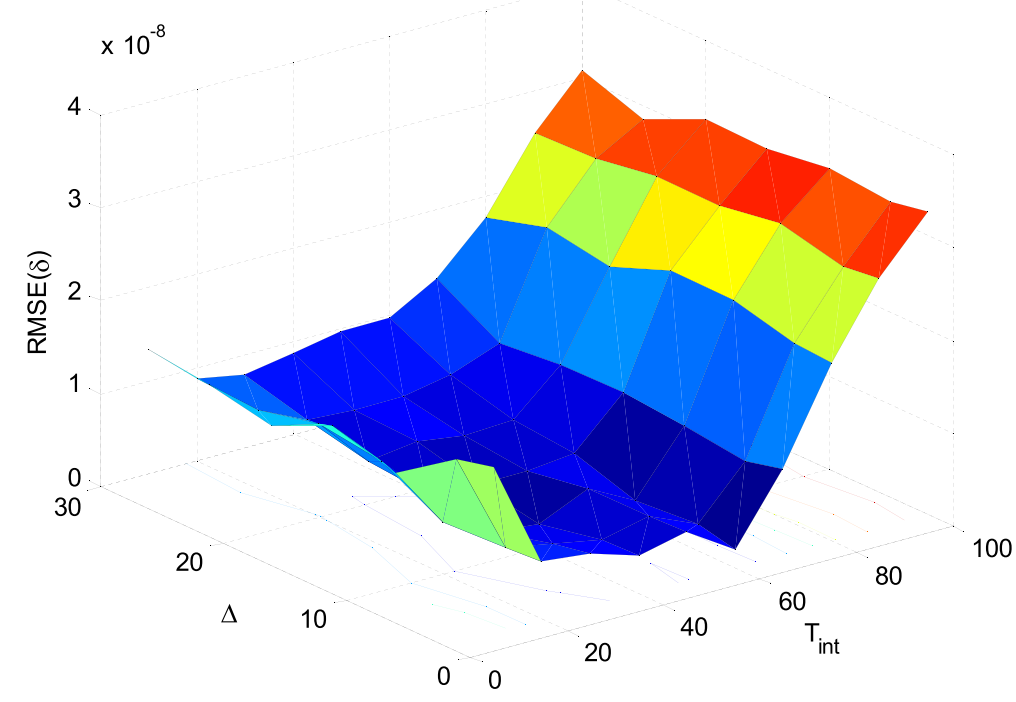

Fig. 9 RMSE of TD energy detection estimator vs. integration window length Tint and integrating step $\Delta$, for NLOS scenario 
We have shown that the time delay can be estimated by the projection of the received signal into two different bases which can be explained physically as a sliding window operation.

We have also investigated on lower bounds of time delay estimation based on the Cramer-Rao lower bound. We have derived analytically the lower bound by using a Karhunen-Loève decomposition of the estimated channel autocorrelation matrix.

We have also seen that the proposed ML TD estimator is impractical (i.e., the ML estimator configuration is based on the optimum unrealizable or noncausal filter). Hence, the choice of a sub-optimum receiver is necessary. Therefore, more practical time delay estimation receivers have recently been proposed. Among all noncoherent UWB receivers, we have chosen the energy detector due to its low complexity implementation.

Hence, in the second part of the paper, we have analyzed the time delay estimation performances with the energy maximization receiver. Simulations results were given using a simulated UWB underground mine channel. This can be considered as the first step for a global positioning system for use mining industry.

\section{Abbreviations}

AWGN: Additive white Gaussian noise; BPF: Band-pass filter; CRLB: CramerRao lower bound; IFI: Inter-frame interference; LOS: Line of sight; MAP: Maximum a posteriori estimator; ML: Maximum likelihood; MMSE: Minimum mean square error; MSE: Mean square error; NLOS: Non line of sight; RMSE: Root mean square error; SNR: Signal-to-noise ratio; TDE: Time delay estimation; UWB: Ultra-wideband; WSN: Wireless sensor networks

\section{Acknowledgments}

The authors would like to thank the School of Electrical Engineering and Computer Science, University of Ottawa for supporting this study.

\section{Funding}

Not applicable.

\section{Availability of data and materials}

Not applicable.

\section{Authors' contributions}

AC developed the theoretical formalism, performed the analytic calculations, and performed the numerical simulations. Both AC, P-MT, and PF contributed to the final version of the manuscript. PF supervised the project. All authors read and approved the final manuscript.

\section{Competing interests}

The authors declare that they have no competing interests.

\section{Publisher's Note}

Springer Nature remains neutral with regard to jurisdictional claims in published maps and institutional affiliations.

\footnotetext{
Author details

'University Ottawa, 800 King Edward Avenue, Ottawa, ON K1N 6N5, Canada.

${ }^{2}$ Department of Electrical and Computer Engineering, Laval University,

Quebec, QC G1V 0A6, Canada. ${ }^{3}$ Sherbrooke University, 2500, boul. de

I'Université, Sherbrooke, QC J1K 2R1, Canada. ${ }^{4}$ Department of Applied

Sciences, University of Quebec in Chicoutimi (UQAC), 555, boul. de

I'Université, Chicoutimi, QC G7H 2B1, Canada.
}

Received: 14 January 2018 Accepted: 22 November 2018

Published online: 10 December 2018

\section{References}

1. V.C. Gungor, G.P. Hancke, Industrial wireless sensor networks: challenges, design principles, and technical approaches. IEEE Trans. Ind. Electron. 56(10), 4258-4265 (2009)

2. C. Shaoyuan, W. Wang, C. Guanrong, Data-aided timing synchronization for FM-DCSK UWB communication systems. IEEE Trans. Ind. Electron. 57(5), 1538-1545 (2010)

3. Z. Cemin, M.J. Kuhn, B.C. Merkl, A.E. Fathy, M.R. Mahfouz, Real-time noncoherent UWB positioning radar with millimeter range accuracy: theory and experiment. IEEE Trans. Microwave Theory Tech. 58(1), 9-20 (2010)

4. N.A. Alsindi, B. Alavi, K. Pahlavan, Measurement and modeling of ultrawideband TOA-based ranging in indoor multipath environments. IEEE Trans. Veh. Technol. 58(3), 1046-1058 (2009)

5. L. Wu, V. Lottici, Z. Tian, Maximum likelihood multiple access timing synchronization for UWB communications. IEEE Trans. Wirel. Commun. 7(11, part 2), 4497-4501 (2008)

6. M.R. Mahfouz, Z. Cemin, B.C. Merkl, M.J. Kuhn, A.E. Fathy, Investigation of high-accuracy indoor 3-D positioning using UWB technology. IEEE Trans. Microwave Theory Tech. 56(6), 13161330 (2008)

7. H.L. Van Trees. Detection, estimation, and modulation theory, part III, Wiley (2002)

8. L. Liping, J.K. Townsend, Near-far resistant synchronization for UWB communications. IEEE Trans. Wirel. Commun. 10(2), 519-529 (2011)

9. S. Gezici, Z. Sahinoglu, H. Kobayashi, H.V. Poor, in Ultra wideband wireless communications, ed. by H. Arslan, Z. N. Chen, M.-G. Di Benedetto. Ultra wideband geolocation, Wiley-Interscience (2006)

10. A. Rabbachin, I. Oppermann, B. Denis, in IEEE 17th international symposium on personal, indoor and Mobile radio Communications. GML ToA estimation based on low complexity UWB energy detection (2006), pp. 1-5

11. A. Rabbachin, I. Oppermann, B. Denis, in Proc. IEEE Int. Conf. on UtraWideband (ICUWB), Waltham, MA. ML time-of-arrival estimation based on low complexity UWB energy detection (2006), pp. 599-604

12. W.-Y. Liu, X.-T. Huang, H. Ding, in Signal Processing (ICSP) 2012 IEEE 11th International Conference on. TOA estimation after power transformation in IR UWB ranging with energy detection receiver, vol 1 (2012), pp. 60-64

13. A. Chehri, P Fortier, P-M Tardif, On the TOA estimation for UWB ranging in complex confined area, 533-536 (2007)

14. A Chehri, P Fortier, Error performance of pulse-based ultra-wideband systems in underground mines, IEEE International Conference on Communications Workshops (ICC), 742-745 (2014)

15. I. Guvenc, Z. Sahinoglu, P.V. Orlik, TOA estimation for IR-UWB systems with different transceiver types. IEEE Trans. Microwave Theory Tech. 54(4), 1876-1886 (2006)

16. A. Giorgetti, M. Chiani, Time-of-arrival estimation based on information theoretic criteria. IEEE Trans. Signal Process. 61(8), 1869-1879 (2013)

17. F. Yin, C. Fritsche, F. Gustafsson, A.M. Zoubir, TOA-based robust wireless geolocation and Cramr-Rae lower bound analysis in harsh LOS/NLOS environments. IEEE Trans. Signal Process. 61(9), 2243-2255 (2013)

18. A. Chehri, P. Fortier, P.-M. Tardif, An investigation of UWB-based wireless networks in industrial automation. IJCSNS 8(2), 179-188 (2008)

19. B. Aziz, A. Nafkha, in Telecommunications (ICT) 2014 21st International Conference on. Implementation of blind cyclostationary feature detector for cognitive radios using USRP (2014), pp. 42-46

20. F. Shang, B. Champagne, I. Psaromiligkos, in IEEE 15th International Workshop on Signal Processing Advances in Wireless Communications (SPAWC). Joint TOA/AOA estimation of IR-UWB signals in the presence of multiuser interference (2014), pp. 504-508

21. A. Chehri, P. Fortier, P.-M. Tardif, Characterization of ultra wideband channel in confined and diffracting rough surface. Wirel. Pers. Commun. (2010). https://doi.org/10.1007/s11277-010-0097-2 